\title{
A novel disinfection protocol using ATP testing for lead garments in the electrophysiology lab
}

\author{
Sahitya Allam ${ }^{1}$ (D) Emily McDowell ${ }^{1} \cdot$ Brittney Heard $^{1} \cdot$ Taelim Kim $^{1} \cdot$ Emmelyn Luu $^{1} \cdot$ Carlene Muto $^{2}$. \\ Sula Mazimba ${ }^{1} \cdot$ Nishaki Mehta $^{3}$
}

Received: 1 July 2020 / Accepted: 28 December 2020 / Published online: 9 January 2021

(C) Springer Science+Business Media, LLC, part of Springer Nature 2021

\begin{abstract}
Purpose Electrophysiology procedures pose infection risk and require surgical room sterility. Currently, there is no universally approved protocol for disinfecting lead garments in the electrophysiology laboratory. This study explores the feasibility of using ATP testing to assess the microbial burden of lead aprons and evaluates the impact of a sanitary intervention.

Methods Adenosine triphosphate (ATP) testing is a well-established hospital standard to quantify biological matter on a surface and, by proxy, the microbial burden. It is measured in RLU (relative light units). Pre-intervention ATP testing was performed on 34 lead garments after use for electrophysiology procedures. The thyroid collar, mid-chest vest, and left axillary areas of the garments were swabbed using a Hygiena SystemSure II luminometer with ATP swabs (Hygiena, Camarillo, CA). These sites were then disinfected with disinfectant wipes (PDI Super Sani-cloth Germicidal Disposable Wipe) and ATP testing was repeated. Results The mean duration of garment wear was $213 \mathrm{~min}$. The thyroid collars had the highest mean RLU before intervention, followed by the mid-chest vest and the left axillary areas. The intervention was found to significantly decrease ATP readings for all three sites ( $p=0.0002, p=0.0001, p=0.0002$ respectively). Linear regression modeling to assess the impact of intervention showed a significant correlation with pre-intervention ATP values for all three sites but no correlation with fluoroscopy time, fluoroscopy dose, or total time spent within the procedure.

Conclusions Lead garments harbor microbial contamination after use according to ATP testing. A sanitary intervention can decontaminate lead garments and potentially reduce rates of hospital infection.
\end{abstract}

Keywords Hospital-acquired infection $\cdot$ Lead garments $\cdot$ ATP testing $\cdot$ Microbial contamination $\cdot$ Surgical sterility

\section{Background}

Infections complicate $1-2 \%$ of cardiovascular implantable electronic device (CIED) procedures, which are expected to become more prevalent due to an aging population with significant cardiovascular morbidities [1]. Ablation procedures, such as for atrial fibrillation, can also be complicated by

Sahitya Allam

sa3py@virginia.edu

Nishaki Mehta

Nishaki.Mehta@beaumont.org

1 Division of Cardiovascular Medicine, University of Virginia Health System, Charlottesville, VA 22903, USA

2 Hospital Epidemiology, University of Virginia Health System, Charlottesville, VA 22903, USA

3 Division of Cardiovascular Medicine, Oakland University William Beaumont School of Medicine, Rochester, MI 48309, USA postoperative infections, including post-procedure sepsis and central venous catheter-related bloodstream infections [2]. Although both types of infections are rare, they can be accompanied by high morbidity, mortality, and medical costs, necessitating the implementation of infection prevention strategies in the electrophysiology laboratory $[1,2]$. Lead garment maintenance may represent a focus for improvement as these garments are often inconsistently sanitized, leading to contamination with multidrug-resistant pathogens [3]. Although these garments are worn under sterile surgical gowns, certain regions of the garments, such as the external surface of the thyroid collar, may not be sufficiently covered by the gown [4]. Intraoperative contact with providers' skin and clothing and perioperative patient-provider interactions are other instances whereby contaminated lead garments can potentially cause infection [4]. Recently, adenosine triphosphate (ATP) testing has emerged as a CDC-approved alternative for assessing microbial contamination as it can provide objective, on-the-spot results $[5,6]$. Testing involves a bioluminescence 
reaction in which the generated amount of light, expressed in relative light units (RLU)/s, is proportional to the amount of ATP present, which serves as a proxy for microbial burden [5]. The method has been validated for assessing surface hygiene in the food industry and is now being utilized in the same capacity for hospital environments [5]. The aim of the present study was to utilize a novel, point-of-care ATP testing protocol for quantifying microbial contamination of lead garments and for measuring the impact of a simple cleaning intervention.

\section{Methods}

Thirty-four lead garments with affixed thyroid collars were tested after use for device implantation procedures and ablation procedures. Each of the lead garments belonged to a unique operator of the electrophysiology lab, with lead garments not being routinely shared among operators. The garments belonged to 24 MDs, 5 RNs, and 5 CVTs. An ATP luminometer was used to approximate the level of microbial contamination (Hygiena SystemSure II with Ultrasnap swab; Hygiena, Camarillo, CA) on the lead garments, which is considered first-generation ATP testing. One hundred RLU was used as the threshold for assessing cleanliness [6]. Cleaning was performed through the use of disinfectant wipes (PDI Super Sani-cloth Germicidal Disposable Wipe; PDI Healthcare, Inc.). These alcohol-based wipes have bactericidal, tuberculocidal, and virucidal properties, making them ideal for targeting the wide array of pathogens colonizing hospital surfaces [7]. Additionally, this wipe has been recognized by the EPA for its protective properties against SARS-CoV-2 [7]. Specific test data is not available, but use of the wipe may cause irritation to the skin or to the respiratory tract if vapors are inhaled at high concentrations [8].

Three locations on the external surfaces of the lead garments were swabbed for $30 \mathrm{~s}$ each: the thyroid collar, midchest vest, and left axillary areas of the chest shield (Fig. 1a). Each swab was inserted into the ATP luminometer system, which provided measurements in RLU within approximately $10 \mathrm{~s}$. Immediately after the procedure, the garments were swabbed to record baseline ATP values (pre-intervention). The established locations were then cleaned with disinfectant wipes (intervention). After disinfection, 3 min were allotted for drying before repeat ATP measurements were taken (postintervention). Total procedure time (time an individual provider spends within a procedure wearing their garment), fluoroscopy time, and fluoroscopy dose were also recorded. Twosample $t$ tests were used to compare mean values of ATP measurements before and after intervention. Differences were reported as significant if $p<0.05$. A linear regression analysis was used to determine the effect of intervention. Analyses were performed using SAS (version 9.4; SAS Institute, Cary, NC).

\section{Results}

The total procedure time ranged from 25 to $428 \mathrm{~min}$, and the mean total procedure time was $212.65 \pm 119.10 \mathrm{~min}$. It was not possible to ascertain the mean duration of wear per procedure across the lifetime of the garments. However, among the procedures included in this study, MDs had significantly longer duration of wear (procedure time) compared to RNs and CVTs $(p=0.0001)$. The thyroid collar registered the largest mean ATP value pre-intervention, followed by the mid-chest vest and left axillary area. ATP values decreased significantly post-intervention for the thyroid collars, vest, and axillary areas of the lead garments $(p=0.0002, p=0.0001, p=$ 0.0002; Fig. 1b). Of the 34 samples of garments, 7 thyroid collars (20.6\%), 6 mid-chest vest shields (17.7\%), and 0 left axillary areas were above the recommended threshold value (100 RLU) prior to disinfection. After the cleaning intervention, 1 thyroid collar (2.9\%), 0 mid-chest vest shields, and 0 left axillary areas failed to fall below the threshold value (Fig. 1b). Additionally, a linear regression model to assess the impact of the cleaning protocol showed a significant correlation with pre-intervention ATP values for all three sites (thyroid $p=0.007$, chest $=0.03$, armpit $=0.008$ ). However, there was no correlation with fluoroscopy time, fluoroscopy dose, or total procedure time. Interestingly, the linear regression model for the intervention for the thyroid collar site demonstrated a significant correlation for MDs compared to RNs and CVTs $(p=0.04)$.

\section{Discussion}

Electrophysiology procedures require the highest practices of surgical sterility [1]. Despite this, there are no standardized disinfection protocols for lead garments in the electrophysiology laboratory. Focusing on lead garment maintenance may prove beneficial in a hospital environment bearing high rates of surface microbial contamination with potentially pathogenic organisms $[3,4,6]$. Overall, our findings suggest that implementing a novel, point-of-care disinfection protocol consisting of ATP testing in conjunction with disinfection wipes may significantly reduce the microbial contamination of lead garments in all three tested regions. However, the thyroid collar region, which may be exposed above the sterile gown, was the most recalcitrant to disinfection of the three, as demonstrated by a thyroid collar failing to drop below the 100 RLU threshold after cleaning. This was likely due to higher microbial burden pre-intervention for thyroid collars, which also led to higher residual burden post-intervention (Fig. 1b). 
Fig. 1 Thyroid collar, mid-chest vest, and left axillary region of a lead garment swabbed for ATP testing (a, in order from left to right), two-sample $t$ test analysis of pre-intervention and postintervention ATP values, also showing \% samples above 100 RLU threshold pre- intervention and post-intervention (b)

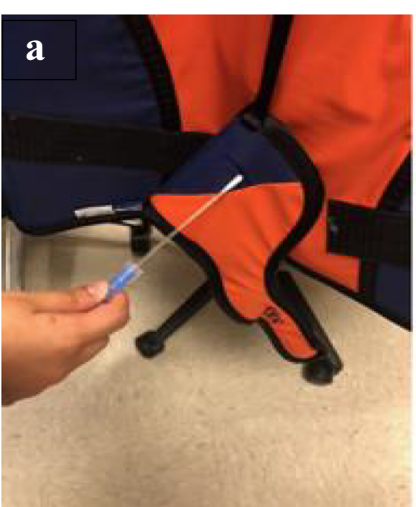

b Region of Lead Garment Swabbed
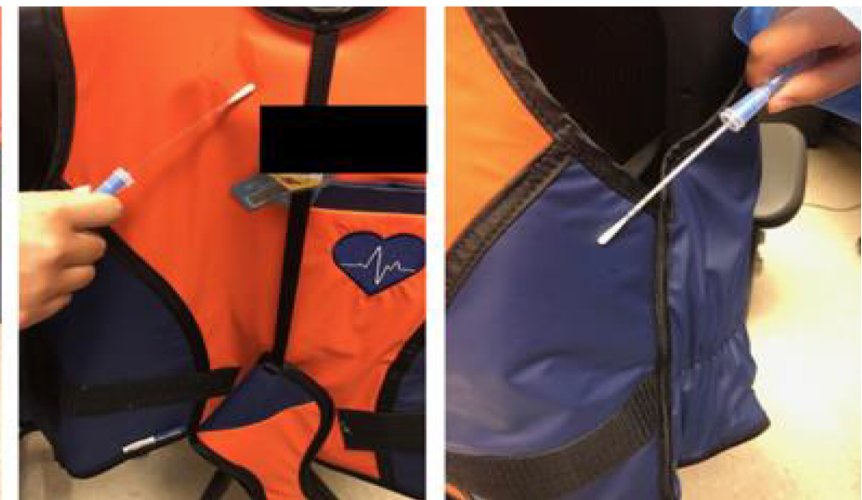

Mean ATP value (RLU) and \% samples above ATP threshold

\begin{tabular}{|l|l|l|l|}
\cline { 2 - 4 } & \multicolumn{1}{|c|}{ Pre-Intervention } & \multicolumn{1}{|c|}{ Post-Intervention } & \multicolumn{1}{c|}{ P-Value } \\
\hline Thyroid Collar & $89.82 \pm 110.90(20.6 \%)$ & $19.38 \pm 41.73(2.9 \%)$ & $0.000190 *$ \\
\hline Mid-chest Vest & $44.21 \pm 50.31(17.7 \%)$ & $7.79 \pm 8.58(0.0 \%)$ & $0.000095 *$ \\
\hline Left Axillary & $14.62 \pm 18.38(0.0 \%)$ & $2.56 \pm 5.01(0.0 \%)$ & $0.000157 *$ \\
\hline
\end{tabular}

A significant correlation between post-intervention thyroid collar ATP values and MD wear suggests that longer, continuous wear of garments by MDs compared to RNs and CVTs results in greater thyroid collar contamination. Nevertheless, our results demonstrate that the cleaning protocol itself was not impacted by procedural elements such as total procedure time (equivalent to duration of garment wear) or fluoroscopy time and dose. Therefore, we suspect that any failure of disinfection around the thyroid collar may not be due to the cleaning protocol but rather the duration of continuous lead garment wear, causing baseline high microbial burden.

We predict that this intervention would be cost-effective and feasible, given the affordability of disinfectant wipes and their widespread availability within hospitals [7]. Comparatively, cardiac device implantation infections cost upwards of $\$ 50,000$ per event [9]. This study is limited by the imperfect correlation between ATP counts and degree of microbial contamination; however, a strict threshold of cleanliness was employed and very low readings were obtained post-intervention, which is associated with low aerobic colony counts [5, 6]. Additionally, ATP testing has been recognized by the CDC as an objective way to appraise cleaning effectiveness in hospital settings and was recommended to us by our Hospital Epidemiology Committee [5].

Further studies are warranted to demonstrate the impact of the cleaning intervention on infection rates. Although, owing to its simplicity and affordability, we believe the current protocol could be reasonably implemented before and after each procedure. We envision a two-pronged approach with a preoperative and postoperative phase. In the preoperative phase, baseline ATP testing is performed on garments for the team members who will scrub for the procedure. If the ATP value is found to be greater than the $100 \mathrm{RLU}$ threshold, disinfectant wipe cleaning must be performed, with a recommended air drying time of $3 \mathrm{~min}$. In the postoperative phase, we always

\section{Pre-op Testing}

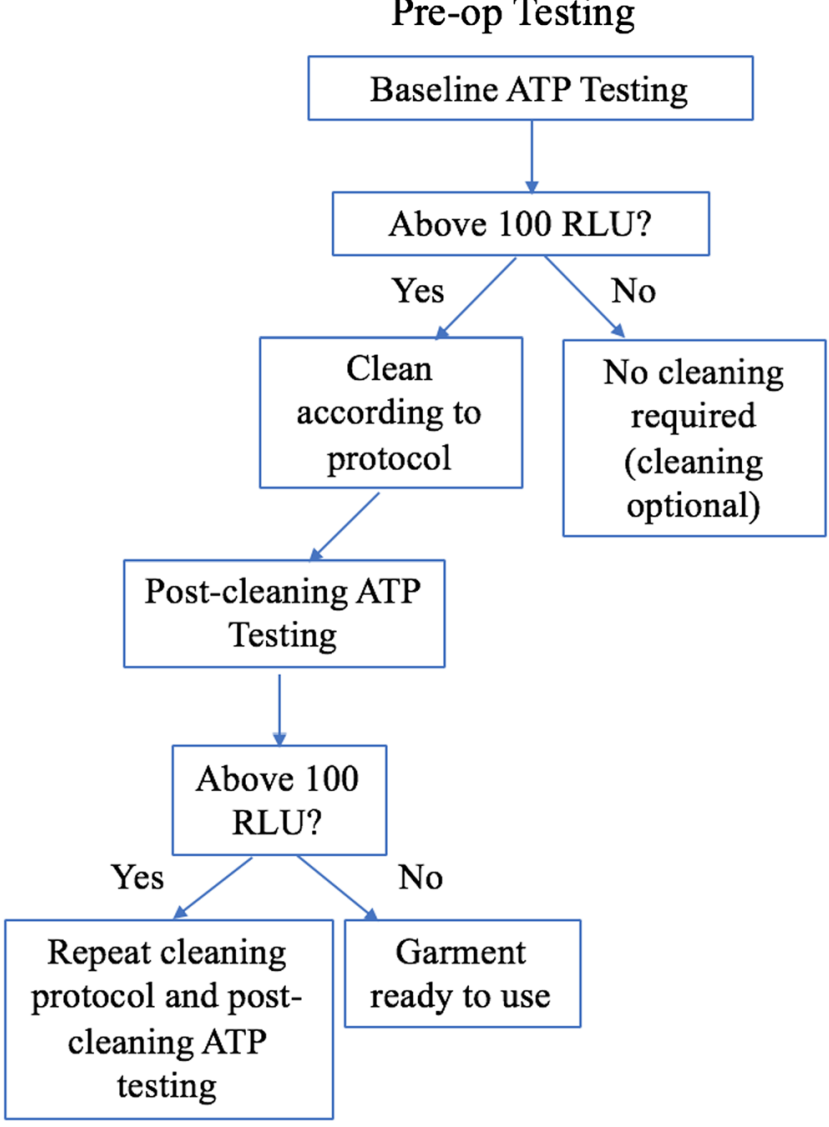

Fig. 2 Preoperative testing flowchart. Cleaning protocol refers to protocol described in the "Methods" section 
recommend cleaning with disinfectant wipes irrespective of ATP testing results. ATP testing is optional in the postoperative phase. The suggested protocol is illustrated in Fig. 2. Either one person can be designated as point of contact for ATP testing and garment cleaning or the individual user can be responsible. This could be guided by individual institutional workflows.

Acknowledgments The authors would like to acknowledge Ashley Taylor and Shaina Sisak for their assistance in the data collection process.

Authors' contributions The principal investigator, Dr. Nishaki Mehta, is a clinical electrophysiologist currently based at William Beaumont Oakland University of Medicine and adjunct faculty at the University of Virginia. She conceived the idea for the study based on her experiences in the various electrophysiology laboratories she has worked at (The Ohio State University, Brigham and Women's Hospital, Harvard Medical School, and the University of Virginia) and while leading the Quality Improvement Committee at The University of Virginia.

Dr. Carlene Muto is an infectious disease physician with extensive knowledge of nosocomial infections and effective disinfection practices. She helped to develop the ATP testing protocol in her role as a leader in the University of Virginia Hospital Epidemiology Department.

Dr. Sula Mazimba is an advanced heart failure specialist at the University of Virginia who performs left and right heart catheterizations where he uses lead-based garments. He offered his expertise to the team.

Dr. Brittney Heard is an electrophysiology fellow at the University of Virginia who helped to guide data collection and data analysis for this study.

Sahitya Allam, Emily McDowell, Taelim Kim, and Emmelyn Luu are University of Virginia medical students who worked under the guidance of the physicians above to collect data for this project, perform preliminary data analysis, and draft the manuscript for publication.

Data availability Data can be made available.

\section{Compliance with ethical standards}

Conflict of interest The authors declare that they have no conflict of interest.
Code availability Not applicable.

\section{References}

1. Tarakji KG, Mittal S, Kennergreen C, et al. Antibacterial envelope to prevent cardiac implantable device infection. N Engl J Med. 2019;381(18):1895-905. https://doi.org/10.1056/nejmc1908355.

2. Deshmukh A, Patel NJ, Pant S, et al. In-hospital complications associated with catheter ablation of atrial fibrillation in the United States between 2000 and 2010. Circulation. 2013;128(19):210412. https://doi.org/10.1161/circulationaha.113.003862.

3. Ang L, Almasoud A, Palakodeti S, Mahmud E. Bacterial contamination of lead aprons in a high-volume cardiac catheterization laboratory and disinfection using an automated ultraviolet-C radiation system. J Invasive Cardiol. 2018;30(11):416-20.

4. Grogan BF, Cranston WC, Lopez DM, Furbee C, Murray CK, Hsu JR. Do protective lead garments harbor harmful bacteria? Orthopedics. 2011. https://doi.org/10.3928/01477447-20110922-09.

5. Options for Evaluating Environmental Cleaning. Centers for disease control and prevention. 2019.https://www.cdc.gov/hai/toolkits/ appendices-evaluating-environ-cleaning.html. Accessed 27 April 2020.

6. Mulvey D, Redding P, Robertson C, et al. Finding a benchmark for monitoring hospital cleanliness. J Hosp Infect. 2011;77(1):25-30.

7. Super Sani-Cloth Germicidal Disposable Wipe. PDI. https://pdihc. $\mathrm{com} /$ products/environment-of-care/super-sani-clothgermicidaldisposable-wipe/. Accessed 27 July 2020.

8. Safety Data Sheet. PDI. 2020. https://pdihc.com/wpcontent/uploads/ 2018/08/SDS-0020-00-English.pdf. Accessed 29 July 2020.

9. Asundi A, Stanislawski M, Mehta P, et al. Development and validation of a semi-automated surveillance algorithm for cardiac device infections: insights from the VA CART program. Sci Rep. 2020;10: 5276. https://doi.org/10.1038/s41598-020-62083-y.

Publisher's note Springer Nature remains neutral with regard to jurisdictional claims in published maps and institutional affiliations. 